\title{
relazioni
}

\section{SESSIONE 6}

\section{MRSA:}

\section{da patogeno ospedaliero a patogeno comunitario}

\author{
Mercoledi 20 Settembre 2006, ore 09.00 - 13.00, Sala BERLINO
}

S6.1

\section{STORIA ED EVOLUZIONE DI MRSA}

\section{Varaldo P.E.}

Istituto di Microbiologia e Scienze Biomediche, Università Politecnica delle Marche, Ancona

Nessuna specie batterica è mai stata segnata da un'antibiotico-resistenza quanto lo Staphylococcus aureus dalla meticillino-resistenza. Quella di MRSA (methicillin-resistant $S$. aureus) è una lunga storia, ormai, che ha inizio 45 anni fa in Gran Bretagna, con i primi sporadici isolamenti poco dopo l'introduzione della meticillina nella pratica clinica. MRSA è da più di un quarto di secolo il patogeno nosocomiale per eccellenza, eppure la sua storia epidemiologica è sempre stata capricciosa: e solo negli ultimi anni, grazie al progresso tecnologico che consente di sequenziare interi genomi e di analizzare vaste popolazioni batteriche con nuove tecniche di tipizzazione altamente discriminative e capaci di indagare le parti più conservate del genoma, si è cominciato a capire qualcosa sulla sua origine, e addirittura a delinearne una storia evolutiva. Se è vero che conosciamo da tempo sia la penicillinbinding protein a bassa affinità (PBP2a) responsabile della meticillino-resistenza sia il gene $(\operatorname{mec} A)$ che la codifica, è un fatto però che solo da poco abbiamo cominciato a conoscere l'elemento cromosomico (SCCmec) di cui il gene mecA fa parte, e a capire come le basi molecolari e genetiche della variabilità di MRSA e di molte delle sue caratteristiche biologiche siano associate all'eterogeneità di tale elemento. Negli ultimi anni stiamo poi assistendo a nuove emergenze, riguardanti MRSA, di impatto potenzialmente epocale, come la comparsa di ceppi clinici altamente resistenti alla vancomicina e la diffusione di MRSA al di fuori del tradizionale contesto ospedaliero, ossia nella comunità. Già c'è chi va preconizzando un nuovo trend per MRSA: in ospedale, ceppi appartenenti a relativamente pochi cloni, sempre più multi-resistenti, anche ai glicopeptidi; e in comunità, ceppi tendenzialmente resistenti solo ai ß-lattamici, ma più eterogenei e potenzialmente anche più patogeni.

\section{S6.2}

\section{LA TIPIZZAZIONE GENICA PER DISCRIMINARE HA-MRSA E CA-MRSA}

\section{Stefani S., Campanile F. \\ Dipartimento di Scienze Microbiologiche \\ Università degli Studi di Catania \\ E-mail: stefanis@unict.it}

In quanto a versatilità di strategie patogene, numero di fattori di virulenza e capacità di sopravvivere e moltiplicarsi in svariati ambienti, S.aureus meticillino-resistente (MRSA) risulta essere il microrganismo più flessibile, responsabile di infezioni gravi acquisite in ambiente ospedaliero e, più recentemente, in comunità. Gli MRSA hanno subito una forte spinta evolutiva, mediata dalla pressione selettiva causata dall'uso di un numero sempre maggiore di agenti antimicrobici.

Sebbene l'applicazione delle metodiche classiche di tipizzazione molecolare (PFGE; RFPLs), insieme ai dati della MLST, abbiano permesso di intraprendere uno studio sulle correlazioni genetiche degli MRSA e predire il genotipo ancestrale dal quale discendono attualmente i cloni maggiormente diffusi, le ricerche relative alle sue basi genetiche sono sempre in continua evoluzione.

L'individuazione in cassette mobili del determinante della resistenza mecA, che codifica la PBP2A a bassa affinità per i ß-lattamici, ha in parte risposto a molti interrogativi riguardo la diffusione della meticillinoresistenza, rimettendo in discussione la presunta origine di MRSA da un unico progenitore; infatti, alla mar- 
cata clonalità si contrappone la variabilità dell'elemento $\mathrm{SCCmec}$, che fa ipotizzare un trasferimento orizzontale realizzatosi all'origine tra diverse linee evolutive ancestrali.

La tipizzazione molecolare degli HA-MRSA ha fornito numerosi dati sulle relazioni intercorrenti tra i cloni più diffusi in ambito ospedaliero e i maggiori cloni internazionali, ma la comparsa di cloni ipervirulenti in comunità, sensibili alla maggior parte di antibiotici non-b-lattamici, con differenti background genetici rispetto a quelli conosciuti, sottolinea la necessità di migliorare e armonizzare i metodi di rilevamento. Inoltre, recentemente nuovi cloni epidemici di MRSA che, paradossalmente, sono resistenti a un numero minore di classi di antibiotici dei cloni precedentemente endemici, si stanno diffondendo in diversi ospedali. $\mathrm{SCCmec}$ in CA-MRSA è di piccole dimensioni, privo di geni per la resistenza oltre a quello per la meticillina, e dotato di ricombinasi funzionali; inoltre, è spesso associato a molteplici geni della virulenza e spesso ai geni della Panton-Valentine leucocidine (PVL).

Ad oggi non esistono studi sulla possibile origine e sulla capacità di diffondere di CA-MRSA, contrastati anche dall'abilità di questi microrganismi di risiedere nella popolazione in modo asintomatico. Solo attraverso l'analisi comparata degli elementi SCCmec e del genotyping è possibile, oggi, mettere in evidenza le origini indipendenti degli H-MRSA e CA-MRSA.

\section{Domande:}

1) Cosa si intende per SCCmec DNA?

a) Una cassetta genica contenente DNA stafilococcico

b) Elementi genetici contenenti almeno il gene mecA

c) Elementi genetici mobili

d) DNA sempre presente in tutti gli stafilococchi

e) Nessuna di queste risposte

2) S.aureus meticillino-resistente "communityacquired" è

a) Più sensibile alla oxacillina

b) Più sensibile agli antibiotici

c) Responsabile di infezioni in ambiente ospedaliero

d) PVL negativo

e) SCCmec positivo

\section{S6.3}

\section{CA-MRSA IN ITALIA E NEL MONDO}

\section{Pantosti A.}

Istituto Superiore di Sanità, Roma

Staphylococcus aureus resistente alla meticillina (MRSA), è stato tradizionalmente considerato un patogeno ospedaliero o comunque associato a pratiche ed ambienti di assistenza sanitaria. Alla fine degli anni ' 90 , si è assistito da una parte ad una più frequente comparsa di infezioni sporadiche da MRSA in individui che non avevano evidenti contatti con l'assistenza sanitaria, dall'altro a vere epidemie in particolari gruppi o comunità "semi-chiuse", quali bambini indianiamericani, bambini che frequentavano asili nido, detenuti, reclute, e componenti di squadre sportive. Le infezioni erano prevalentemente cutanee, ma anche insolitamente gravi. I ceppi MRSA isolati da queste infezioni, definiti CA-MRSA (cioè community-acquired o associated MRSA), apparivano diversi dai ceppi classici ospedalieri per caratteristiche fenotipiche e/o genotipiche. I ceppi CA-MRSA sono in genere sensibili ad antibiotici diversi dai beta-lattamici, hanno un profilo genetico che ne caratterizza l'appartenenza a cloni diversi da quelli a cui appartengono MRSA ospedalieri e tipicamente contengono l'elemento di resistenza SCCmec di tipo IV. Inoltre spesso producono la tossina di Panton-Valentine (P-V), che è considerata un fattore di virulenza favorente $\mathrm{i}$ fenomeni necrotici.

I dati sulla prevalenza e circolazione di CA-MRSA sono spesso confusi e discordanti a causa delle diverse definizioni adottate. In molti studi, soprattutto retrospettivi, la definizione è basata solo su criteri epidemiologici (presunta acquisizione dell'infezione in comunità oppure colonizzazione al momento dell'ospedalizzazione). In questi casi, soprattutto se non viene effettuata un'anamnesi accurata per escludere precedenti contatti del paziente, diretti o indiretti, con l'assistenza sanitaria, si rischia di sovrastimare la quota di CA-MRSA. Quando si seguono anche criteri microbiologici, si può descrivere con più accuratezza la quota di infezioni o colonizzazioni dovuta a ceppi con caratteristiche tipiche di CA-MRSA. In una metanalisi di studi condotti in varie parti del mondo, in prevalenza in Nord America ed in Europa, la frequenza di infezioni attribuite a CA-MRSA in pazienti ospedalizzati, sulla base di criteri esclusivamente epidemiologici, era del 30-37\%. Tuttavia, la maggior parte dei pazienti presentava uno o più fattori di rischio per l'acquisizione di MRSA ospedalieri. Studi più recenti, in cui sono state prese in esame anche le caratteristiche fenotipiche e/o genotipiche dei ceppi, hanno dimostrato che $<1 \%$ dei soggetti in comunità senza fattori di rischio sono portatori di CA-MRSA. Questi ceppi sono in genere sensibili ad antibiotici diversi dai beta-lattamici ma non tutti posseggono SCCmec di tipo IV, e soprattutto non posseggono tossina di P-V.

Caratteristica è invece l'associazione tra tipici CAMRSA ed infezioni: se si prendono in considerazione manifestazioni infettive insorte in comunità, quali infezioni cutanee in bambini o polmoniti gravi in individui giovani, allora i ceppi responsabili sono tipici CAMRSA con SCCmec di tipo IV e tossina P-V. Per complicare il quadro, tipici CA-MRSA possono essere introdotti in ospedale e causare epidemie, come ad esempio tra neonati in reparti di neonatologia. I ceppi CA-MRSA, con SCCmec di tipo IV e P-V positivi, appartengono dal punto di vista genetico ad un nume- 